\title{
ANÁLISE COMPARATIVA DE DESEMPENHO DE REDES RESIDENCIAIS IEEE 802.11N E HOMEPLUG AV
}

\author{
E. L. Trindade', A. L. P. S. Campos ${ }^{2}$ e R. C. O. Moreira ${ }^{3}$ \\ ${ }^{1}$ Faculdade Natalense para o Desenvolvimento do Rio Grande do Norte \\ ${ }^{2}$ Universidade Federal do Rio Grande do Norte, Departamento de Engenharia de Comunicações \\ ${ }^{3}$ Universidade Potiguar, Engenharia de Computação \\ E-mail: cpd@piramidenatal.com.br ${ }^{1}$, antonio.luiz@cnpq.br ${ }^{2}$, rilco.eng@gmail.com ${ }^{3}$
}

Artigo submetido em fevereiro/2012 e aceito em dezembro/2013

\section{RESUMO}

Este artigo apresenta uma análise comparativa dos padrões $802.11 \mathrm{n}$ e Homeplug AV, abordando melhorias desses padrões com relação aos seus anteriores. São realizados testes do desempenho de cada padrão em ambiente residencial, considerando como

parâmetro analisado a taxa de transmissão. 0 interesse foi determinar 0 desempenho relativo destas tecnologias, considerando a taxa de transmissão em função da distância de transmissão, do tamanho dos pacotes e do número de nós ativos na rede.

PALAVRAS-CHAVE: IEEE 802.11n, Homeplug AV, Taxa de transmissão, Rede de Computadores.

\section{COMPARATIVE ANALYSIS OF PERFORMANCE OF RESIDENTIAL NETWORKS IEEE 802.11N AND HOMEPLUG AV}

\section{ABSTRACT}

This paper presents a comparative analysis of HomePlug AV and $802.11 \mathrm{n}$ standards, addressing improvements to these standards with respect to its predecessors. Performance tests are standard in every home environment, considering parameter analyzed the

transmission rate. The interest was to determine the relative performance of these technologies, considering the transmission rate depending on transmission distance, the size of packets and the number of active nodes in the network.

KEYWORDS: IEEE 802.11n, Homeplug AV, Throughput, Computer network. 


\section{ANÁLISE COMPARATIVA DE DESEMPENHO DE REDES RESIDENCIAIS IEEE 802.11N E HOMEPLUG AV}

\section{INTRODUÇÃO}

Tecnologias de rede candidatas para prestação de serviços de redes SOHO (Smal Office Home Office) convenientes e largamente espalhadas podem ser categorizadas como redes sem fio, redes com fio e redes sem novos fios. Para redes residenciais as tecnologias mais apropriadas são o padrão 802.11 e o padrão Homeplug.

O padrão 802.11 tem sido usado com sucesso, especialmente o $802.11 \mathrm{~g}$ por ter se tornado padrão de mercado. Já o padrão HomePlug não é tão utilizado como o padrão 802.11 , mas ele aparece como uma tecnologia alternativa e competitiva em relação ao padrão 802.11 , especialmente em casas com mais de um pavimento. A principal norma em uso é o HomePlug 1.0 .

Embora bem-sucedidos, esses padrões não podem atender as novas aplicações multimídia que requerem altas taxas de transmissão. Aplicações como áudio/vídeo e jogos multijogadores exigem maior largura de banda, suporte QoS (Quality of Service) e taxas de transmissão de até $20 \mathrm{Mbps}$, bem como limites superiores sobre parâmetros de QoS tais como latência, jitter e perda de pacotes.

Devido a isso, novos padrões foram desenvolvidos recentemente. A força-tarefa 802.11 criou o padrão 802.11n baseados em tecnologia MIMO (Multiple Input Multiple Output) e com melhoramentos nas camadas física e de enlace. A HomePlug Alliance, por sua vez, criou o padrão HomePlug AV (Audio/Video). Ambos os padrões visam taxas que possam suportar aplicações multimídia.

O objetivo principal deste trabalho é realizar um estudo de desempenho no mundo real dos recursos de tecnologia de redes IEEE 802.11n e HomePlug AV. Nosso interesse é determinar o desempenho relativo destas tecnologias, considerando a taxa de transmissão em função da distância de transmissão, do tamanho dos pacotes e do número de nós transmitindo.

\section{PADRÃO IEEE 802.11N}

O IEEE, após o sucesso do padrão $802.11 \mathrm{~g}$, montou uma força tarefa no sentido de desenvolver um novo padrão 802.11, com o objetivo de oferecer velocidades maiores do que a transmissão atual de redes cabeadas a $100 \mathrm{Mbps}$, e melhorias no que diz respeito a faixa de confiabilidade, transmissão e latência. Então, o novo padrão IEEE 802.11n surgiu.

O padrão IEEE 802.11n é o resultado da mudança e melhorias nos algoritmos de transmissão e utilização de uma nova tecnologia MIMO, que permite que a placa de rádio use vários fluxos de transmissão de dados em paralelo, utilizando vários conjuntos de transmissores, receptores e antenas. Na prática, o que acontece é que um processador de sinal compara as diferentes sequências de dados e usa a informação para preencher as lacunas de alguns dos outros que sofreram degradação no meio [HAUGDAHL, 2007].

O padrão IEEE 802.11n promete aumento na velocidade de transmissão e na banda passante. Segundo [HAUGDAHL, 2007], a velocidade nominal aumentou de 54 Mbps para taxas 
que variam de 150 a $600 \mathrm{Mbps}$, e os equipamentos $802.11 \mathrm{n}$ mais sofisticados, como é o caso de equipamentos de transmissão $4 \times 4$, capazes de transmitir quatro fluxos simultâneos usando múltiplos fluxos, tornam a banda do sinal quase duas vezes maior.

\subsection{CAMADA FÍSICA (PHY)}

O principal objetivo da força tarefa IEEE 802.11n era definir um padrão que melhorasse a máxima taxa de dados para atingir, no mínimo, 100 Mbps na camada de enlace (MAC) e permitisse a coexistência com os outros padrões em uso. Para isso, foram elaboradas algumas proposições para a camada física, tais como: uso da tecnologia MIMO (Multiple Input Multiple Output), multiplexação por divisão de frequências ortogonais (OFDM) e vários esquemas de canais com diferentes larguras de banda [SKORDOULIS et al. 2008].

Para alcançar altas taxas de transmissão, o 802.11n combina uma série de melhorias na camada física. A primeira é a redução do intervalo entre as transmissões, ou intervalo de guarda, de 800 ns para 400 ns, que fornece um ganho de aproximadamente $11 \%$ em sua taxa de transmissão. Houve também um aumento no número de subportadoras de dados de 48 para 52 portadoras, resultando em um ganho proporcional na taxa de transmissão. Somando os dois com uma melhoria no algoritmo de transmissão de erros, foi possível chegar a uma taxa de transmissão de 72,2 Mbps, utilizando um único canal. Assim, o 802.11n ofereceria um pequeno ganho de 33\% em relação ao padrão 802.11g [Air Magnet 2008].

A principal melhoria da camada PHY do padrão $802.11 \mathrm{n}$ é o uso de múltiplas antenas transmissoras/receptoras nas estações. O uso da tecnologia MIMO feito pelo $802.11 \mathrm{n}$ combina vários rádios e dois canais de fluxo simultâneos, podendo ser de dois ou quatro fluxos, duplicando ou quadruplicando a taxa de dados, chegando a 144,4 e 288,8 Mbps, respectivamente. Para isso, o padrão 802.11 n define diferentes configurações de antenas (MxN), com combinações de $2 \times 2,2 \times 3,3 \times 3$ e $4 \times 4$ antenas. Isso se refere ao número de antenas transmissoras (M) e receptoras (N) [XIA, HAMDI AND LETAIEF 2009].

Outro recurso opcional do padrão $802.11 \mathrm{n}$, mas que beneficia apenas os novos dispositivos, são os canais de $40 \mathrm{MHz}$ que usam duas vezes mais largura de banda para dobrar a taxa de transmissão. Para atingir $288,8 \mathrm{Mbps}$, utilizando apenas dois fluxos, é utilizado o sistema HT40, que usa dois canais simultaneamente, ocupando uma faixa de freqüência de $40 \mathrm{MHz}$. Um ponto de acesso que combina o uso do HT40 com quatro rádios dobraria a taxa teórica, chegando a $600 \mathrm{Mbps}$. $\mathrm{O}$ grande problema é que uma faixa de $40 \mathrm{MHz}$ corresponde a quase toda a faixa de frequência usada por redes $802.11 \mathrm{~g}$, o que acentua o problema já crônico de interferência de redes nas proximidades. Antecipando isso, o padrão 802.11n oferece também o uso da banda de $5 \mathrm{GHz}$ que pode ser usada para reduzir esse problema [XIA, HAMDI AND LETAIEF 2009].

A compatibilidade com os clientes $802.11 \mathrm{~g}$ e $802.11 \mathrm{~b}$ foi mantida, de modo que é possível migrar gradualmente. A observação principal é que a combinação de clientes $802.11 \mathrm{n}$ e $802.11 \mathrm{~g} / \mathrm{b}$ reduz o desempenho da rede, embora o percentual varie bastante de acordo com o modelo utilizado.

O padrão 802.11 n define 77 permutações possíveis de fatores que determinam a taxa de dados. Um inteiro simples define o esquema de Modulação e Codificação (MCS) atribuído a cada permutação de modulação, taxa de codificação, intervalo de guarda, largura do canal e número 
de fluxos espaciais. O padrão 802.11n pode utilizar técnicas de modulação diferentes como: BPSK (Binary PSK), QPSK (Quadrature PSK) e diferentes níveis de QAM (Quadrature Amplitude Modulation). Podem ser usadas diferentes técnicas de modulação e taxas de codificação em cada fluxo espacial [XIA, HAMDI AND LETAIEF, 2009].

\subsection{CAMADA DE ENLACE (MAC)}

As melhorias físicas descritas até agora aumentam ao máximo a taxa de dados, mas fazem uso ineficiente do espectro eletromagnético, sem as melhorias da camada MAC do padrão $802.11 n$.

A primeira melhoria é a confirmação de bloco. A confirmação de bloco reduz o número de ACKs que o receptor deve enviar ao transmissor para confirmar a entrega do quadro. Transmissores 802.11a/g esperam um ACK para cada quadro não multicast/broadcast, mas transmissores 802.11n também aceitam ACKs de blocos, que confirmam o recebimento de vários frames unicast. Por exemplo, em vez de enviar 8 ACKs para confirmar os quadros de 1 a 8 , um receptor 802.11n pode dizer a mesma coisa com apenas um ACK de bloco [HAUGDAHL, 2007].

Outra melhoria da camada MAC é a agregação de quadros. Este recurso aumenta a carga que pode ser transmitida por cada frame 802.11 , reduzindo a sobrecarga da camada MAC de $83 \%$ para menos de $58 \%$ (usando um MSDU) e 14\% (quando se usa um MPDU). Dispositivos $802.11 \mathrm{a} / \mathrm{g}$ podem enviar mais de 2.304 bytes de carga por quadro, mas os novos dispositivos $802.11 \mathrm{n}$ têm a opção de agrupamento para a transmissão de quadros em conjunto, aumentando o tamanho da carga para reduzir o overhead causado pelo espaçamento inter-frame e o preâmbulo [SKORDOULIS et al. 2008]. Há duas opções de agregação:

- A agregação em grupo do MAC Service Data Unit (A-MSDU) de pacotes da LLC (Logical Link Control - MSDUs) com o 802.11e, independentemente da origem ou destino. 0 resultado do quadro MAC contém um cabeçalho MAC, seguido por até 7935 bytes MSDU.

- A agregação do MAC Protocol Data Unit (A-MPDU) ocorre mais tarde, após os cabeçalhos MAC serem adicionados a cada MSDU. Completados os quadros MAC (MPDUs) eles são então agrupados em cargas PHY de até 65535 bytes.

Outras modificações incluem: Qualidade de Serviço (QoS), para suportar aplicações sensíveis a atrasos, como Voz sobre WLAN (VoWLAN) e streaming multimídia, economia de energia (ou PSMP), economia de bateria para dispositivos WLAN portáteis; mudança de largura de banda de canal; melhor gestão dos recursos rádio, suporte para roaming rápido, dentre outros.

\section{PADRÃO HOMEPLUG AV}

Redes de computadores baseadas no padrão HomePlug 1.0 estão disponíveis em todo o mundo. Este padrão é adequado para aplicações como acesso à Internet, mas não pode suportar algumas aplicações de multimídia, porque essas aplicações exigem maior largura de banda com qualidade de serviço (QoS). Por exemplo, MPEG2 de alta definição (HDTV) necessita de até 24 Mbps de taxa de transmissão, bem como limites superiores sobre os parâmetros de QoS tais como latência, jitter e perda de pacotes [AFKHAMIE et al. 2005]. 
Cenários para uso de redes residenciais típicas incluem suporte simultâneo para múltiplos streams de multimídia. Assim, redes residenciais devem ser capazes de fornecer taxas de transmissão variando entre $50 \mathrm{Mbps}$ a $60 \mathrm{Mbps}$. A HomePlug Powerline Alliance lançou um novo padrão o HomePlug AV (HPAV), que se destina a apoiar estas novas aplicações multimídia. Nesta seção, discutimos as camadas física (PHY) e de enlace (MAC) do HPAV.

\subsection{CAMADA FÍSICA (PHY)}

A Camada Física (PHY) do HomePlug AV (HPAV) utiliza multiplexação por divisão de frequências ortogonais em janelas (Windowed Ortoghonal Frequency Division Multiplex WOFDM), que usa uma janela não retangular para melhorar a filtragem das portadoras. O HPAV usa símbolos OFDM longos com 1.155 portadoras, das quais apenas 917 são utilizáveis, em conjunto com um intervalo de banda de guarda flexível, operando na faixa de frequência de 2 $\mathrm{MHz}$ a $28 \mathrm{MHz}$ [HOMEPLUG ${ }^{\circledR}$ POWERLINE ALLIANCE 2005].

Os símbolos WOFDM geram formas de onda das sub-portadoras que são altamente concentradas em frequência, permitindo assim a criação eficiente de espaços no espectro simplesmente desligando portadoras OFDM. Por exemplo, espaços de $30 \mathrm{~dB}$ para as bandas de rádio amador são obtidos com facilidade e eficiência pela desativação de portadoras OFDM, sem a necessidade de uma filtragem. Este recurso fornece ao sistema a flexibilidade para satisfazer diferentes exigências regulatórias em todo o mundo [ATHEROS 2009].

Visando a redução das retransmissões o HPAV implementa algumas técnicas de correção de erro intensivas (Forward Error Correction - FEC). Para manter interoperabilidade com o padrão HomePlug 1.0, o HPAV usa os códigos convolucionais e Reed-Solomon. Além disso, o HPAV também usa códigos turbo convolucionais (TCC), uma moderna técnica que usa dois ou mais códigos convolucionais em paralelo juntos com um espaçador. Isso fornece robustez de desempenho em torno de 0,5 dB de capacidade do canal de Shannon [HOMEPLUG ${ }^{\circledR}$ POWERLINE ALLIANCE 2005].

Para modular as portadoras OFDM, o HPAV utiliza várias técnicas de modulação como: DBPSK, DQPSK e QAM. As modulações DBPSK e DQPSK garantem compatibilidade com o padrão HomePlug 1.0. A constelação QAM varia de acordo com o meio de transmissão. Na medida em que o ambiente fica mais ruidoso ou o número de colisões aumenta, a quantidade de bits por símbolo diminui, a fim de garantir uma maior robustez na transmissão. Essa variação de bits por símbolo vai de 3 bits/símbolo/portadora (8-QAM) a 10 bits/símbolo/portadora (1024-QAM) [KATAR et al. 2006].

Através desse novo padrão é possível a transmissão de dados a uma taxa de até 200 Mbps. A camada física utiliza esses $200 \mathrm{Mbps}$ para prover uma taxa de $150 \mathrm{Mbps}$ de dados na sua camada MAC, o que mostra uma eficiência maior que o HomePlug 1.0 na relação de taxa de transmissão MAC/PHY.

\subsection{CAMADA DE ENLACE (MAC)}

O HPAV é capaz de fornecer uma disputa orientada a conexão, o que garante os requisitos de qualidade de serviço (QoS) para áudio, vídeo e dados. Essa contenção de serviço baseia-se em TDMA distribuindo slots de tempo adequados para suportar as demandas de QoS necessárias para uma conexão e tecnologia CSMA/CA, que é aplicado sobre o tráfego com níveis de 
prioridade, que remove os dados de baixa prioridade durante um breve período de resolução de prioridade no início da janela de contenção [HOMEPLUG ${ }^{\circledR}$ POWERLINE ALLIANCE 2005].

Para fornecer serviços de comunicação eficaz, HPAV implementa uma arquitetura flexível, administrada pelo coordenador central (CCo). O CCo fornece um período de sinalização, que é um tempo de estabilização do TDMA e CSMA/CA. O período de sinalização está dividido em três regiões: região de sinais; região CSMA e região livre de disputa (Contention Free - CF) [KATAR et al. 2006].

O período de sinalização é sincronizado com o ciclo de corrente alternada, proporcionando estabilidade. Isso também proporciona uma melhor adaptação para a sincronização de canais de interferência, resultando em uma melhora na taxa de transmissão. 0 período de sinalização fornece uma estimativa de quando os próximos períodos de sinalização ocorrerão (Persistent Beacon), permitindo que as comunicações não sejam perdidas, caso a estação perca alguma região de sinalização [HOMEPLUG ${ }^{\circledR}$ POWERLINE ALLIANCE 2005].

A camada MAC fornece serviços com contenção (CSMA) e serviços livre de contenção (CF), com suas respectivas regiões durante a sinalização. A região de persistência livre de contenção (Persistence Contention Free - PCF) permite que o HPAV forneça os requisitos de QoS garantidos da camada superior (High Layer Entity - HLE). A HLE utiliza as especificações de conexão (CSPEC) para especificar os seus requisitos de QoS. O gerenciador de conexão (Connection Manager - CM) da estação avalia o CSPEC e, se possível, comunica os requisitos necessários do CCo e pede a ele uma alocação livre de disputa. As características de QoS especificadas no CSPEC incluem: garantia de banda, latência fixa, jitter, dentre outros [HOMEPLUG ${ }^{\circledR}$ POWERLINE ALLIANCE 2005].

O HPAV usa um esquema de enquadramento de dois níveis MAC. As MSDUs recebidas são empacotadas com sobrecarga mínima em quadros MAC, formando um fluxo de quadros MAC. Este é tratado como um fluxo de bytes pelo processo de segmentação, que gera segmentos de tamanho fixo para a transmissão confiável. A cada segmento é dado um cabeçalho e um rastreador que permitem que ele seja criptografado, enviado, decifrado e entregue de forma independente como um bloco PHY (PB). O cabeçalho PB contém números de sequência que permitem a remontagem do fluxo de quadros MAC original a partir de segmentos entregues fora de ordem. Ele também contém informações que permite ressincronização após perda de uma parcela do fluxo de quadros MAC. Em cada PB é enviado seu próprio bloco de FEC e a sequência de checagem PB permite que blocos FEC incorrigíveis sejam detectados de forma confiável. Cada PB pode ser reconhecido individualmente usando uma resposta de reconhecimento seletivo e só os PBs danificados são retransmitidos [AFKHAMIE 2005].

\section{CENÁRIOS DE TESTES}

Para realizar a análise de desempenho dos padrões 802.11n e HomePlug AV, foram efetuados testes de campo, durante trinta dias, aproximadamente, em uma residência de tamanho médio com aproximadamente $80 \mathrm{~m}^{2}$. A Figura 1 ilustra a planta baixa da residência utilizada nos testes. 


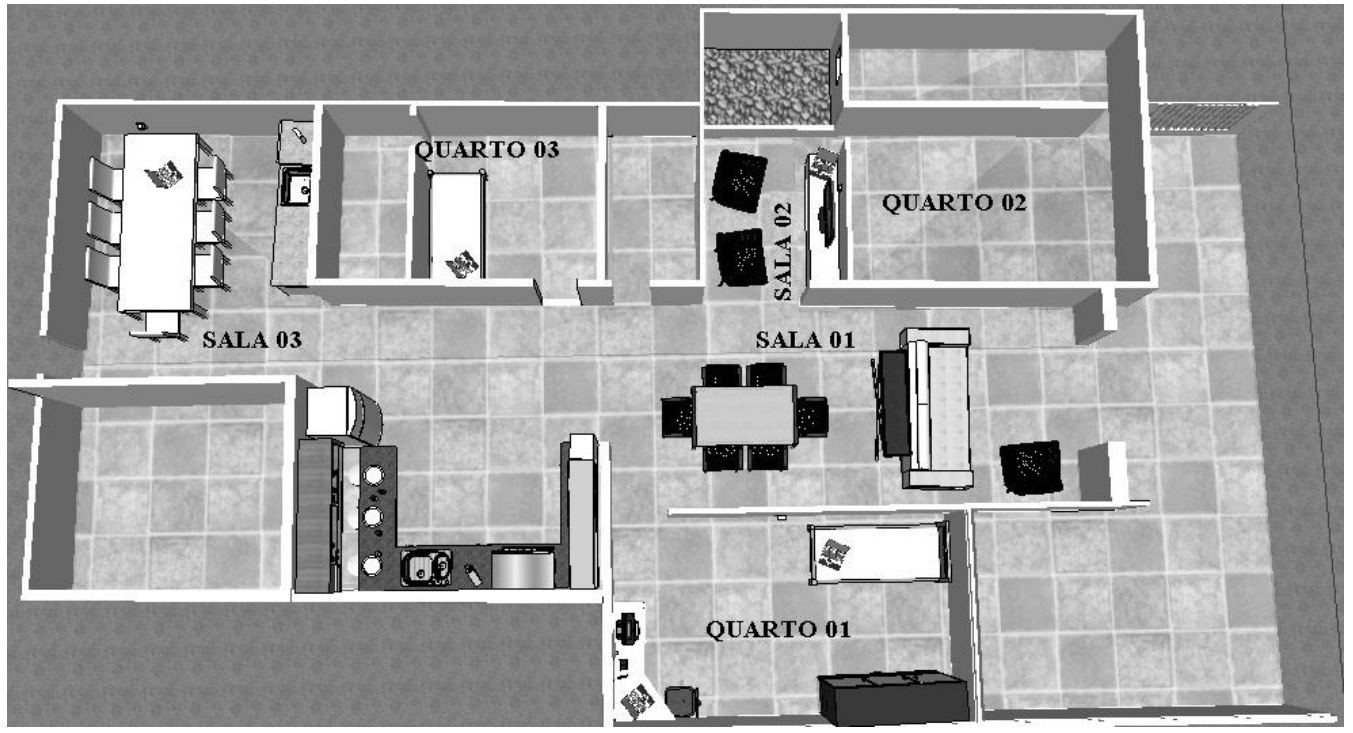

Figura 1 - Planta baixa da residência utilizada nos testes.

Os equipamentos usados nos testes foram os seguintes:

- Microcomputador com processador Pentium 4, 4 GB de RAM, HD 320 GB, placa de rede 10/100 Mbps e placa PCI 802.11n com 3 antenas (encore) 802.11n.

- Note Book, processador Intel Dual Core, Memória RAM de 4 GB, HD 320 GB, placa de rede 10/100/1000 Gbps, placa Wireless 802.11n.

- Pontes Homeplug AV: Adaptador de rede Ethernet Homeplug da Aztech HL110E, taxa de transmissão de até $200 \mathrm{Mbps}$, criptografia AES de 128 bits.

Para testar o padrão HPVA o microcomputador e o notebook foram conectados através da linha de energia por meio de duas pontes Homeplug AV. Para os testes do $802.11 \mathrm{n}$ foi mplementada uma rede sem fio de modo típico infra-estruturada. Neste modo, todos os nós sem fios se comunicam entre si através de um ponto de acesso e compartilham a largura de banda em dois saltos.

\section{METODOLOGIA DOS TESTES E RESULTADOS}

A taxa de transmissão foi medida para diferentes distâncias entre as estações móveis dentro da casa. O microcomputador foi localizado no Quarto 01 (Figura 1) e o notebook foi posicionado em diferentes cômodos da casa.

As medições foram realizadas de forma sucessiva, obtendo-se a taxa de transmissão de cada transmissão realizada. Em todos os testes realizados, para medir a taxa de transmissão na camada de aplicação, foi utilizado o software gratuito o PLC Test. Esse software permite variar o tamanho dos arquivos transmitidos e efetuar transmissões simultâneas, simulando diversos nós ativos na rede.

Foram considerados quatro cenários de testes em uma residência monofásica. 0 tamanho dos arquivos transmitidos variou entre $800,1600,2400,3200,4000,4800,5600,6400,7200 \mathrm{~KB}$ e $8000 \mathrm{~KB}$, sendo realizadas 90 transmissões para cada arquivo, totalizando 810 transmissões, referentes a 30 dias de teste. Para manter a coerência, em todos os testes, a distância entre os 
dois pontos se manteve constante. Os testes foram realizados em diferentes turnos do dia. Foram consideradas quatro cenários de teste, de uma tomada para a outra dentro do Quarto 01, do Quarto 01 para a Sala 01, do Quarto 01 para a Sala 02 e do Quarto 01 para o Quarto 03; as distâncias entre os nós foram de, aproximadamente, 2, 5, 7 e $10 \mathrm{~m}$, respectivamente.

A Figura 2 ilustra os testes obtidos para o padrão 802.11 n considerando as quatro diferentes distâncias. Para curtas distâncias $(2 \mathrm{~m})$ a taxa de transmissão oscilou em torno de 45 Mbps. Para as distâncias 2 e 3 a taxa ficou próxima de $41 \mathrm{Mbps}$. Variando um pouco mais a distância $(10 \mathrm{~m})$, nós observamos uma queda na taxa de transmissão considerável, assim como um aumento na variação dessa taxa. $\mathrm{O}$ arquivo usado nesse teste foi de $8000 \mathrm{~KB}$.

A Figura 3 ilustra os testes obtidos para o padrão HPAV considerando as mesmas distâncias utilizadas nos testes apresentados na Figura 2. Para curtas distâncias $(2 \mathrm{~m})$ a taxa de transmissão ficou próxima daquela obtida para o padrão $802.11 \mathrm{n}$, entretanto, quando a distância aumenta, nós observamos uma variação maior na taxa de transmissão para o padrão HPAV. Para os cenários 2 e 3 a taxa de transmissão sofreu uma redução de 40\%, mantendo-se quase igual nas duas situações. Variando um pouco mais a distância $(10 \mathrm{~m})$, nós observamos uma queda na taxa de transmissão de mais de $60 \%$. Um fato interessante é que enquanto a taxa de transmissão passou a oscilar mais com o aumento da distância para o padrão 802.11n, para o padrão HPAV ela se manteve mais constante com o aumento da distância. $O$ arquivo usado nesse teste foi de $8000 \mathrm{~KB}$.

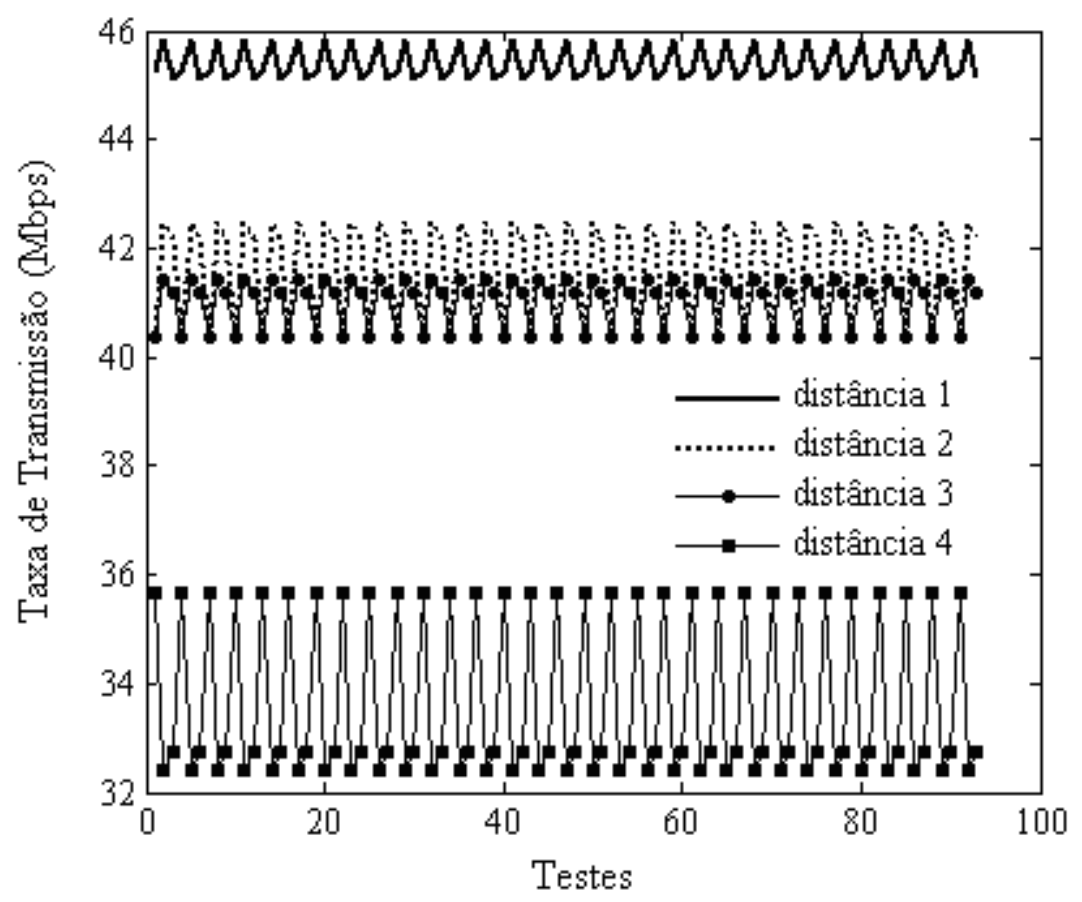

Figura 2 - Resultados dos testes para o padrão 802.11n, considerando quatro diferentes distâncias. 


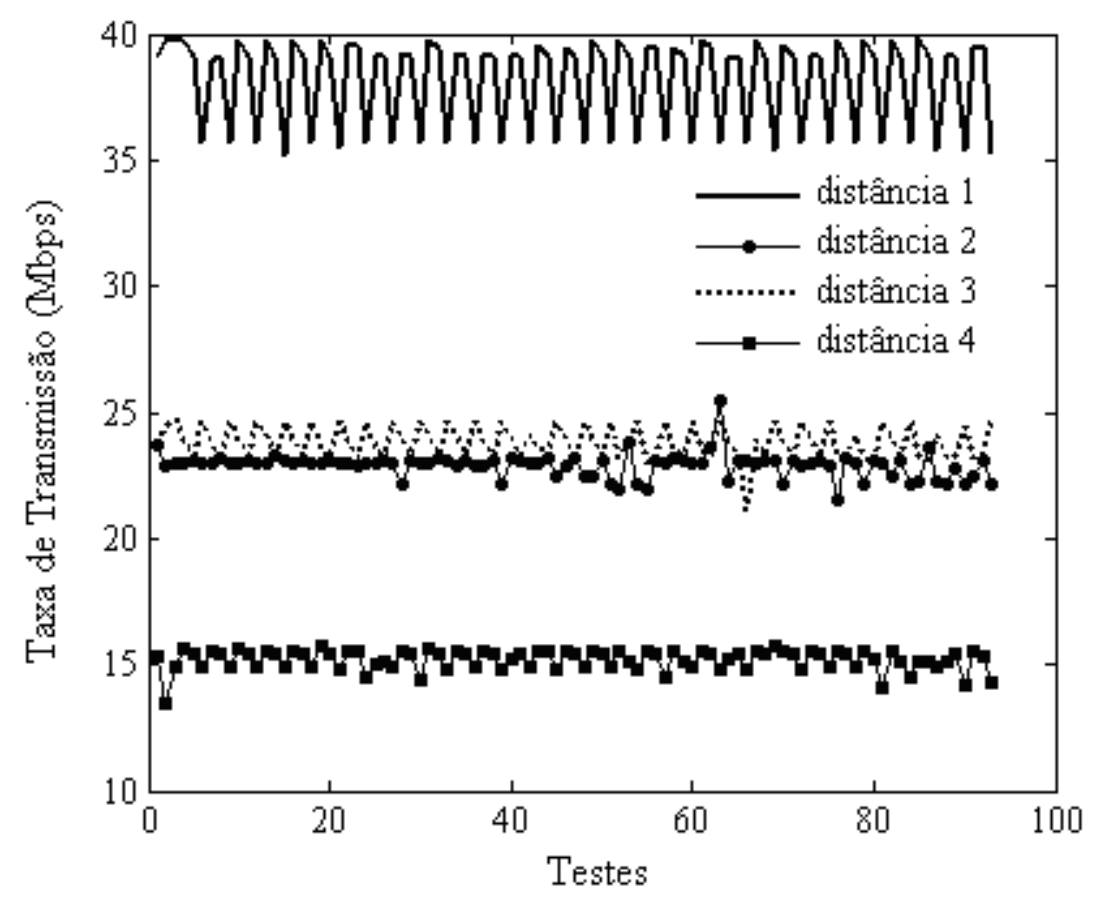

Figura 3 - Resultados dos testes para o padrão HPAV, considerando quatro diferentes distâncias.

A Tabela 1 resume esses resultados. Podemos ver o aumento na variação da taxa de transmissão para o padrão 802.11n pelo desvio padrão calculado em cada cenário. O contrário é observado para o HPAV. Outro fato é que a vazão média do HPAV é próxima da vazão média do 802.11n para a distância 1, mas fica em torno de 50 \% para as demais. Isso mostra uma forte influência da distância no padrão HPAV.

Tabela 1: Resultados para os diferentes cenários e padrões.

\begin{tabular}{|l|c|c|c|c|c|}
\hline \multicolumn{1}{|c|}{ CENÁRIO } & \multirow{2}{*}{$\begin{array}{c}\text { DISTÂNCIA } \\
(\mathrm{m})\end{array}$} & $\begin{array}{c}\text { Média } \\
\text { (Mbps) }\end{array}$ & $\begin{array}{c}\text { Desvio } \\
\text { Padrão }\end{array}$ & $\begin{array}{c}\text { Média } \\
\text { (Mbps) }\end{array}$ & $\begin{array}{c}\text { Desvio } \\
\text { Padrão }\end{array}$ \\
\hline $\begin{array}{l}\text { 1 - Tomada no Quarto 01 para a outra tomada } \\
\text { no mesmo quarto }\end{array}$ & 2 & 38,17 & 1,75 & 45,41 & 0,32 \\
\hline 2-Quarto 01 para a Sala 01 & 5 & 22,90 & 0,50 & 41,64 & 0,99 \\
\hline 3-Quarto 01 para a Sala 02 & 7 & 23,82 & 0,65 & 40,99 & 0,46 \\
\hline 4-Quarto 01 para o Quarto 03 & 10 & 15,19 & 0,42 & 33,62 & 1,46 \\
\hline
\end{tabular}

A Figura 4 ilustra os testes obtidos para os padrões HPAV e 802.11 considerando o Cenário 1 de transmissão e usando 1 e 4 nós ativos. O padrão 802.11n teve uma pequena redução na taxa de transmissão caindo de aproximadamente $45 \mathrm{Mbps}$ para aproximadamente 40 Mbps. Já o padrão HPAV sofreu uma forte influência caindo de aproximadamente $38 \mathrm{Mbps}$ para aproximadamente $17 \mathrm{Mbps}$, uma redução de mais de $50 \%$ na taxa de transmissão média. 0 arquivo usado nesse teste foi de $8000 \mathrm{~KB}$. 


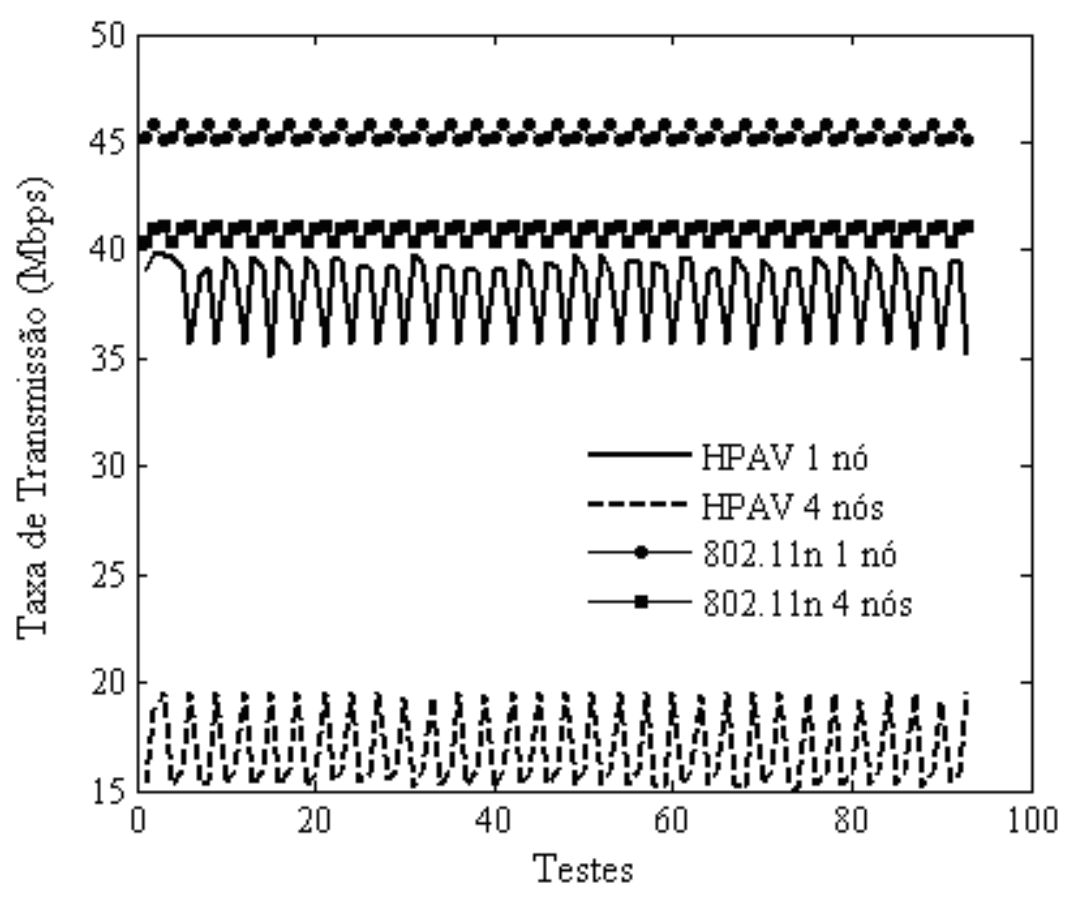

Figura 4 - Resultados dos testes para os padrões 802.11n e HPAV, considerando um e quatro nós ativos.

A Tabela 2 resume os resultados dos testes. Podemos observar ainda que a taxa de transmissão do 802.11n ficou próxima da média tanto para 1 quanto para 4 nós, enquanto que para o padrão HPAV essa taxa oscilou bastante em torno da média, pelos desvios padrões calculados.

Tabela 2: Resultados para 1 e 4 nós ativos para os diferentes padrões..

\begin{tabular}{|c|c|c|c|c|}
\hline \multirow{2}{*}{ NÚMEOR DE NÓs } & \multicolumn{2}{|c|}{ HPAV } & \multicolumn{2}{c|}{$802.11 n$} \\
\cline { 2 - 5 } & $\begin{array}{c}\text { Média } \\
\text { (Mbps) }\end{array}$ & $\begin{array}{c}\text { Desvio } \\
\text { Padrão }\end{array}$ & $\begin{array}{c}\text { Média } \\
\text { (Mbps) }\end{array}$ & $\begin{array}{c}\text { Desvio } \\
\text { Padrão }\end{array}$ \\
\hline 1 & 38,17 & 1,75 & 45,41 & 0,32 \\
\hline 4 & 16,89 & 1,89 & 40,90 & 0,32 \\
\hline
\end{tabular}

A Figura 5 ilustra os testes obtidos para os padrões HPAV e 802.11 considerando o Cenário 1 de transmissão, usando 1 nó ativo e variando o tamanho dos pacotes transmitidos. 0 padrão 802.11n sofreu mudanças consideráveis nas médias da taxa de transmissão indo de aproximadamente $51 \mathrm{Mbps}$ para aproximadamente $45 \mathrm{Mbps}$. Já o padrão HPAV manteve sua taxa média de transmissão praticamente constante mantendo essa taxa em torno de $39 \mathrm{Mbps,}$ aproximadamente. 


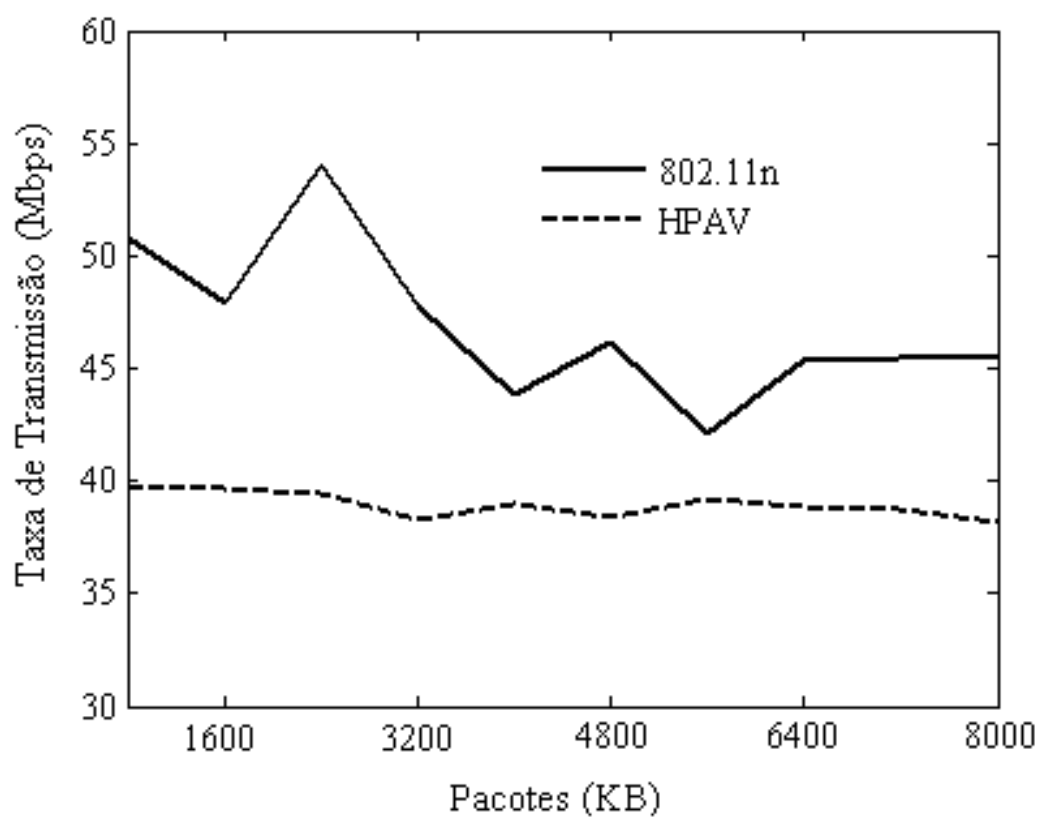

Figura 5 - Resultados dos testes para os padrões 802.11n e HPAV em função do tamanho dos pacotes.

A Tabela 3 resume os resultados dos testes. Podemos observar ainda que a taxa de transmissão do padrão HPAV ficou próxima da média tanto para todos os tamanhos de pacotes, enquanto que para o padrão $802.11 \mathrm{n}$ essa taxa oscilou bastante principalmente para pacotes curtos.

Tabela 3: Resultados para diferentes tamanhos de pacotes e padrões.

\begin{tabular}{|c|c|c|c|c|}
\hline \multirow{2}{*}{ PACOTES (Kbps) } & \multicolumn{2}{|c|}{ HPAV } & \multicolumn{2}{c|}{$802.11 n$} \\
\cline { 2 - 5 } & $\begin{array}{c}\text { Média } \\
\text { (Mbps) }\end{array}$ & $\begin{array}{c}\text { Desvio } \\
\text { Padrão }\end{array}$ & $\begin{array}{c}\text { Média } \\
\text { (Mbps) }\end{array}$ & $\begin{array}{c}\text { Desvio } \\
\text { Padrão }\end{array}$ \\
\hline 800 & 39,63 & 0,36 & 50,81 & 6,62 \\
\hline 100 & 39,68 & 0,26 & 47,90 & 1,12 \\
\hline 1600 & 39,45 & 0,46 & 54,00 & 1,51 \\
\hline 3200 & 38,31 & 0,15 & 47,81 & 3,34 \\
\hline 4000 & 38,97 & 0,40 & 43,80 & 1,90 \\
\hline 4800 & 38,44 & 1,80 & 46,11 & 0,39 \\
\hline 5600 & 39,17 & 0,48 & 42,16 & 0,03 \\
\hline 6400 & 38,89 & 0,54 & 45,39 & 0,11 \\
\hline 7200 & 38,73 & 0,94 & 45,41 & 0,32 \\
\hline 8000 & 38,17 & 1,75 & 45,41 & 0,32 \\
\hline
\end{tabular}

\section{CONCLUSÕES}

Neste trabalho, foram descritas as camadas físicas e de enlace dos padrões $802.11 \mathrm{n}$ e Homeplug AV. Foram implementadas redes wireless e redes Homeplug AV em uma residência média. Foi medido o desempenho dessas redes considerando-se apenas suas respectivas taxas de transmissão. Diversas situações foram analisadas, considerando-se diferentes distâncias de 
transmissão, quantidade nós ativos na rede e tamanho dos pacotes transmitidos. Para a medição da taxa de transmissão, foi utilizado o software gratuito PLC Test, da INNOVUS.

Os testes mostraram que o padrão 802.11 n sofre menos influência com a distância e com o número de nós ativos do que o HPAV. Com relação ao tamanho dos pacotes, o HPAV praticamente não apresentou variações, enquanto que o $802.11 \mathrm{n}$ sofreu variações. $O$ padrão 802.11 mostrou-se apropriado para novas aplicações de multimídia, mantendo taxas de transmissão superiores a $20 \mathrm{Mbps}$ em todos os testes realizados. Já o HPAV apresentou taxas inferiores a $20 \mathrm{Mbps}$ para distâncias maiores que $10 \mathrm{~m}$ e 4 nós ativos de rede, podendo ser inadequado para algumas aplicações de multimídia.

Por fim, este trabalho apresentou uma avaliação inédita de redes HomePlug AV e 802.11n no que diz respeito ao seu desempenho considerando a taxa de transmissão. Como complementação deste trabalho, sugere-se a realização de testes residenciais considerando outros parâmetros de desempenho, tais como atraso e perda de pacotes.

\section{REFERÊNCIAS BIBLIOGRÁFICAS}

1. AFKHAMIE, K. H., KATAR, S., YONGE, L., and NEWMAN, R., "An Overview of the upcoming HomePlug AV Standard", International Symposium on Power Line Communications and Its Applications, pp. $400-404,2005$.

2. AIR MAGNET, "802.11n Primer", White Paper, 2008.

3. ATHEROS POWERLINE, "HomePlug ${ }^{\circledR}$ AV Technology Overview", White Paper, 2009.

4. HAUGDAHL, J. S., "Inside 802.11n Wireless LANs, Practical Insights and Analysis", BitCricket LLC, White Paper, 2007.

5. HOMEPLUG ${ }^{\circledR}$ POWERLINE ALLIANCE, “HomePlug AV White Paper”, 2005.

6. KATAR, S., KRISHNAM, M., NEWMAN, R. and LATCHMAN, H., "Harnessing the potential of powerline communications using the HomePlug AV standard", Broadband Technology, pp. 16 $-26,2006$.

7. SKORDOULIS, D. et al., "IEEE 802.11n MAC Frame Aggregation Mechanisms for NextGeneration High-Throughput WLANs", IEEE Wireless Communications, pp. 40-47, 2008.

8. XIA, Q., HAMDI, M., and LETAIEF, K. B., “Open-Loop Link Adaptationfor Next-Generation IEEE 802.11n WirelessNetworks", IEEE Transactions on Vehicular Technology, Vol. 58, No. 7, pp. $3713-3723,2009$. 